\title{
Overweight children with type 1 diabetes have a more favourable lipid profile than overweight non-diabetic children
}

\author{
Mariska van Vliet • Josine C. van der Heyden • Michaela Diamant • \\ Inès A. von Rosenstiel • Roger K. Schindhelm • Martijn W. Heymans • \\ Desiderius P. M. Brandjes • Jos H. Beijnen • Henk J. Aanstoot • Henk J. Veeze
}

Received: 31 May 2011 / Accepted: 6 September 2011 /Published online: 4 October 2011

(C) The Author(s) 2011. This article is published with open access at Springerlink.com

\begin{abstract}
In the present article, we aimed to compare the cardiometabolic risk between overweight children with and without type 1 diabetes (T1DM). Therefore, data with regard to cardiometabolic risk parameters of 44 overweight Caucasian children (3-18 years) with T1DM were matched with 44 overweight peers without T1DM for sex, ethnicity, age and standard deviation score of BMI (Z-BMI). Detailed history was taken, information regarding anthropometrics and family history were collected and blood pressure was measured. Blood samples were collected for evaluation of lipid profiles (fasting in controls, non-fasting in T1DM children), alanine aminotransferase and $\mathrm{HbAlc}$ (in children with T1DM). It was found that overweight children with T1DM had lower median standard deviation score of waist circumference (Z-WC) as compared to the overweight control
\end{abstract}

M. van Vliet and J. C. van der Heyden contributed equally

M. van Vliet $(\bowtie) \cdot$ I. A. von Rosenstiel

Department of Pediatrics, Slotervaart Hospital,

Louwesweg 6,

1066 EC Amsterdam, The Netherlands

e-mail: m.vanvliet@slz.nl

M. van Vliet $\cdot$ M. Diamant

Diabetes Center/Department of Internal Medicine,

VU University Medical Center,

De Boelelaan 1117

1081 HV Amsterdam, The Netherlands

J. C. van der Heyden · H. J. Aanstoot $\cdot$ H. J. Veeze

Diabeter,

Haringvliet 72,

3011 TG Rotterdam, The Netherlands group [median, 2.0 (interquartile range, IQR, 1.5-2.3) vs. 2.6 (IQR, 2.0-2.9), $P<0.001]$. After adjustment for $\mathrm{Z}-\mathrm{WC}$, in children with T1DM, median high-density lipoprotein cholesterol levels were significantly higher and median low-density lipoprotein cholesterol lower in T1DM children, as compared to their peers without T1DM [1.40 (IQR, 1.21.5 ) vs. 1.2 (IQR, 1.0-1.3) and 2.7 (IQR, 2.5-3.2) vs. 3.0 (IQR, 2.5-3.4), respectively, all $P<0.01]$. When dividing children according to glycaemic status, children with suboptimal glycaemic control had higher values of triglycerides as compared to well-controlled children [1.3 (IQR, $1.0-1.8$ ) vs. 0.96 (IQR, $0.80-1.2$ ), $P=0.036$ ]. In conclusion, overweight children with T1DM have a more favourable lipid profile, as compared to non-diabetic overweight controls, in spite of a higher frequency of a positive family

\footnotetext{
R. K. Schindhelm

Department of Clinical Chemistry, Isala Clinics,

Dr C.A. van Heesweg 2 ,

8025 AB Zwolle, The Netherlands

M. W. Heymans

Department of Methodology and Applied Biostatistics, Institute of Health Sciences, VU University Medical Center, De Boelelaan 1118,

1081 HV Amsterdam, The Netherlands

D. P. M. Brandjes

Department of Internal Medicine, Slotervaart Hospital,

Louwesweg 6,

1066 EC Amsterdam, The Netherlands

J. H. Beijnen

Pharmacy \& Pharmacology, Slotervaart Hospital,

Louwesweg 6,

1066 EC Amsterdam, The Netherlands
} 
history of CVD, T2DM and hypertension. Still, paediatricians should give extra attention to cardiometabolic risk factors within this vulnerable group, taking into account the already high cardiometabolic risk.

Keywords Paediatric $\cdot$ Obesity . Overweight $\cdot$ Risk factors for cardiovascular disease Type 1 diabetes

\section{Introduction}

In line with the global obesity epidemic, an increasing proportion of children becomes overweight and may develop additional cardiometabolic risk factors, including dyslipidaemia, high blood pressure and glucometabolic disorders $[19,23]$. Unless children are encouraged to lose weight successfully, many cardiometabolic risk factors that emerge during childhood persist throughout adulthood and may ultimately lead to premature death $[3,5,11]$. Although the effect of overweight has been studied extensively in the general population, the effect of weight gain on cardiometabolic health in individuals with type 1 diabetes (T1DM) remains largely unknown. According to the SEARCH for Diabetes in Youth study, a higher proportion of children with T1DM are overweight (but not obese) as compared to non-diabetic controls, suggesting co-occurrence of different mechanisms, which lead to weight gain in subjects with T1DM [9]. Next to a sedentary lifestyle, other factors may highly promote weight gain in T1DM patients, such as intensified insulin therapy to optimise glycaemic control [1]. Although there is a lack of studies investigating the relationship of causes of weight gain (i.e., intensified insulin treatment for glycaemic control vs. sedentary lifestyle) with the development of cardiometabolic risk factors, it has been reported in the Diabetes Control and Complications Trial (DCCT) that the development of overweight in individuals with T1DM caused similar adverse changes in cardiometabolic risk [14]. As these cardiometabolic changes have their onset in childhood, they will promote the process of atherosclerosis in both nondiabetic children and children with T1DM. Interestingly, however, the latter group tends to develop more severe atherosclerotic lesions starting earlier in life as compared to non-diabetic controls [15]. In a previous study, we demonstrated that both overweight non-diabetic children and children with T1DM have a high prevalence of cardiometabolic risk factors. As compared to their normal weight peers with T1DM, overweight children without T1DM had a higher prevalence of the metabolic syndrome, isolated hypertension and high alanine aminotransferase (ALT) [22]. In the present study, we compare overweight children with and without T1DM, with respect to cardiometabolic risk variables, after matching for sex, age, standard deviation score of BMI (Z-BMI) and ethnicity.

\section{Materials and methods}

Selection of T1DM and non-T1DM children

In 2007 and 2008, data from 283 children with T1DM, who visited a specialised clinic for paediatric diabetes care (Diabeter), were collected for cross-sectional analysis for cardiometabolic risk factors, as described previously [22]. From this data set $(n=99$; Z-BMI, -2.5 to 4.1$)$, all Caucasian children with overweight were selected and matched according to sex, age, ethnicity (i.e. being Caucasian) and Z-BMI to non-diabetic children derived from a data set ( $n=750$; Z-BMI, 1.4-4.2) from a paediatric obesity clinic (Slotervaart Hospital) in Amsterdam, The Netherlands. Children of the latter group were excluded from the study when using glucose- or lipid-lowering drugs, corticosteroids (chronically) or drugs acting on the central nervous system. Additionally, children with (suspected) syndromes, T1DM, type 2 diabetes (T2DM) or secondary causes of obesity, such as hypothyroidism, hypogonadism and pituitary disorders, were excluded [21]. In total, 44 successful matches could be made.

\section{Definitions}

Overweight was classified using standardised scores of BMI (Z-BMI), according to the Dutch Fourth National Growth Study, with a cut-off value for overweight being a $\mathrm{Z}-\mathrm{BMI} \geq 1.1$ [6]. A detailed history was taken [including family history for T2DM, premature cardiovascular disease (CVD) and hypertension according to the criteria of the American Diabetes Association] [2] and anthropometrics were recorded. Three consecutive blood pressure measurements were performed at the non-dominant arm, with at least 1-min interval, in the seated position and after $10 \mathrm{~min}$ of rest. The mean of these three measurements was used in the analyses. Furthermore, blood samples were drawn for evaluation of lipid profiles and ALT. This was done in the non-fasting state (due to both ethical and practical reasons) in children with T1DM [22] and in the fasting state in children from the control group [21]. Since lipid levels were performed in the non-fasting state in children with T1DM and fasting state in children without T1DM, analysis were performed after recalculation of lipids in case lipids were non-fasting (in children with T1DM). One of the reasons for the decision of recalculation of non-fasting values instead of fasting values is because the Friedewald formula for calculation of low-density lipoprotein (LDL) 
cholesterol is more reliable when applied on fasting state values [7]. As reported in a recent study investigating fasting and non-fasting lipid levels in children with T1DM, mean variation was $0 \mathrm{mmol} / \mathrm{L}$ for high-density lipoprotein (HDL) cholesterol, $+0.11 \mathrm{mmol} / \mathrm{L}$ for total cholesterol and $-0.31 \mathrm{mmol} / \mathrm{L}$ for triglycerides [16]. After recalculation of aforementioned lipid profiles in children with T1DM, LDL cholesterol was calculated with the Friedewald formula [7]. Since the calculation of LDL cholesterol in T1DM patients has been shown to be less reliable, nonHDL cholesterol was calculated as well [17]. In children with T1DM, additional data on insulin regimen, diabetes duration and HbAlc were collected. Target levels for $\mathrm{HbA} 1 \mathrm{c}$ were defined as $\mathrm{HbA} 1 \mathrm{c}<7.5 \%$ [18]. A positive family history was defined as a family history positive for CVD (i.e. occurring before 55 years of age), T2DM and/or hypertension in a first or second degree family member [2].

\section{Laboratory analyses}

In children with T1DM, non-fasting lipids and ALT were measured in the non-fasting state by enzymatic methods on the Hitachi Cobas C501 analyser (Roche, Mannheim, Germany) with an intra- and inter-assay coefficient of variation of $<2.1 \% \mathrm{HbA} 1 \mathrm{c}$. HbAlc was measured by a immunochemical assay with intra- and inter-assay coefficients of variation of $<3.7 \%$ and $<4.3 \%$, (reference range, 4-6\%). In the non-diabetic subjects, plasma glucose, ALT and lipids were determined with SYNCHRON LX 20 (Beckman Coulter, Galway, Ireland), with intra-assay coefficients of variation (CV) of $\leq 3.5 \%$ for ALT and $\leq 3 \%$ for plasma glucose and fasting lipids. LDL cholesterol was calculated with the Friedewald formula [7]. Plasma insulin levels were measured by an immunoluminometric assay (Immulite 200 system, DPC, Los Angeles, CA, USA; intraassay variation, 3-6\%; inter-assay variation, 3-5\%).

\section{Statistical analysis}

Data are shown as median [interquartile range (IQR)]. Using power analysis, a sample size of 45 pairs was needed to detect a difference of 0.3 for LDL cholesterol (SD, 0.8; power, 80\%; alpha, 0.05) and 0.15 for HDL cholesterol (SD, 0.5; power, 80\%; alpha, 0.05). Differences between case and control groups were tested with generalised estimating equations models (GEEs), with cardiometabolic variables, and family history as dependent variables. GEE instead of 'paired analysis' was used since it allows adjustment for confounders. Results were presented with and without adjustment for Z-WC. For comparing prevalence rates of positive family history between groups,
Fisher exact tests were used. In determining differences for cardiometabolic risk within the T1DM group (stratified for glycaemic status, $\mathrm{HbAlc}<7.5$ versus $\geq 7.5 \%$ ), independent Student's $t$ tests were performed. All analyses were performed with SPSS 16.0 for windows.

\section{Results}

Baseline characteristics

In total, 44 overweight T1DM children could be matched to 44 non-diabetic children. Overall, median age was 12.0 (IQR, 9.1-15.6) years, and median Z-BMI was 2 (IQR, 1.9-2.6). In the T1DM group, median diabetes duration was 5.85 (IQR, 2.97-9.16) years, and median insulin dose of 0.96 (IQR, 0.69-1.34) IU/kg/day. Thirty-two children used insulin pump therapy, while multiple insulin injection therapy was used by ten children, three children were treated with a single or two insulin injections. In children with T1DM, median fasting glucose levels were 5.1 (IQR, 4.9-5.3) $\mathrm{mmol} / \mathrm{L}$, and median fasting insulin was 94 (IQR, 69-126) $\mathrm{pmol} / \mathrm{L}$.

Differences in cardiometabolic risk factors between overweight children with and without T1DM

Table 1 lists the cardiometabolic characteristics of the 44 T1DM children and the 44 children without T1DM and presents recalculated lipid levels for total cholesterol, triglycerides and LDL cholesterol. Median measured cholesterol levels in T1DM children were 4.3 (IQR, 3.94.7) $\mathrm{mmol} / \mathrm{L}$ for total cholesterol and 1.2 (IQR, 0.9-1.6) $\mathrm{mmol} / \mathrm{L}$ for triglycerides. Since $\mathrm{Z}-\mathrm{WC}$ was significantly lower in children with T1DM [2.0 (IQR, 1.5-2.3) vs. 2.6 (IQR, 2.0-2.9), $P<0.001]$, results with and without adjustment for Z-WC are shown. For both LDL and non-HDL cholesterol, both recalculated and measured lipid levels were significantly lower in children with T1DM than in children without T1DM (all $P<0.05$ ). No significant differences were found between groups for total cholesterol and triglycerides; however, HDL cholesterol was significantly higher in children with T1DM, relative to nondiabetic children.

The role of glycaemic control in children with T1DM

When T1DM children were divided according to glycaemic control, 31 had suboptimal control (HbAlc $\geq 7.5 \%)$ and 13 had good glycaemic control (HbAlc $<7.5 \%)$. These groups did not differ with respect to age and diabetes duration. Children with suboptimal glycaemic control had higher 
Table 1 Cardiometabolic variables in overweight children with T1DM as compared to non-diabetic children

\begin{tabular}{|c|c|c|c|c|}
\hline & T1DM overweight & Non-T1DM overweight & $P$ value & $P$ value $^{\mathrm{a}}$ \\
\hline$N$ & 44 & 44 & & \\
\hline Sex, boys, $n(\%)$ & 15 & 15 & - & - \\
\hline Age (years) & $12.0(9.1-15.8)$ & $12.1(9.2-15.1)$ & - & - \\
\hline Age $>11$ years, $n(\%)$ & 18 & 17 & - & - \\
\hline BMI $\left(\mathrm{kg} / \mathrm{m}^{2}\right)$ & $23.3(20.8-27.0)$ & $25.0(21.7-28.0)$ & - & - \\
\hline Z-BMI & $2.3(1.9-2.6)$ & $2.4(2.1-2.7)$ & - & - \\
\hline Waist & $77.8(69.0-88.0)$ & $86.5(72.5-96.0)$ & $<0.001$ & $<0.001$ \\
\hline Z-WC & $2.0(1.5-2.3)$ & $2.6(2.0-2.9)$ & $<0.001$ & - \\
\hline Systolic blood pressure $(\mathrm{mmHg})$ & $118(104-127)$ & $112(110-123)$ & 1.00 & 0.20 \\
\hline Diastolic blood pressure (mmHg) & $69(65-74)$ & $70(66-75)$ & 0.04 & 0.14 \\
\hline Total cholesterol $(\mathrm{mmol} / \mathrm{L})^{\mathrm{b}}$ & $4.3(3.9-4.7)$ & $4.6(4.0-4.9)$ & 0.30 & 0.16 \\
\hline HDL cholesterol (mmol/L) & $1.3(1.1-1.4)$ & $1.2(1.0-1.3)$ & $<0.001$ & $<0.001$ \\
\hline LDL cholesterol $(\mathrm{mmol} / \mathrm{L})^{\mathrm{b}}$ & $2.7(2.5-3.2)$ & $3.0(2.5-3.4)$ & 0.011 & 0.01 \\
\hline Triglycerides $(\mathrm{mmol} / \mathrm{L})^{\mathrm{b}}$ & $0.9(0.6-1.5)$ & $0.9(0.6-1.5)$ & 0.80 & 0.69 \\
\hline non-HDL cholesterol $(\mathrm{mmol} / \mathrm{L})^{\mathrm{b}}$ & $2.9(2.7-3.5)$ & $3.5(2.8-3.9)$ & 0.03 & 0.05 \\
\hline ALT (IU/L) & $20(15-24)$ & $21(16-27)$ & 0.49 & 0.63 \\
\hline
\end{tabular}

Tested with generalised estimating equations model. Data expressed as median (interquartile range). To convert values for cholesterol to millimoles per liter, multiply by 0.02586 . To convert values for triglycerides to millimoles per liter, multiply by 0.01129

$Z$ - $B M I$ Standard deviation score of BMI, $Z$ - $W C$ standard deviation score of waist circumference, $A L T$ alanine aminotransferase, $H D L$ high-density lipoprotein, $L D L$ low density lipoprotein

${ }^{a}$ Models additionally adjusted for Z-WC

${ }^{\mathrm{b}}$ Recalculated values from non-fasting state in T1DM children

values of (non-fasting) triglycerides, LDL cholesterol and non-HDL cholesterol as compared to well regulated children: 1.3 (IQR, 1.0-1.8) vs. 0.96 (IQR, 0.80-1.18) $\mathrm{mmol} / \mathrm{L}$ for triglycerides, 2.8 (IQR, 2.5-3.6) vs. 2.6 (IQR, $2.4-2.7$ ) mmol/ L for LDL cholesterol and 3.0 (IQR, 2.8-3.8) vs. 2.7 (IQR, 2.5-2.8) $\mathrm{mmol} / \mathrm{L}$ for non-HDL cholesterol, all $P<0.05$. Blood pressure and ALT levels were similar across the two T1DM groups.

Prevalence of a positive family history

Figure 1 shows the prevalence of a positive family history of CVD, T2DM, hypertension and hypercholesterolaemia. Overall, a positive family history for any of aforementioned diseases was present in 34 children with T1DM, against 10 in the non-T1DM overweight group $(P<0.001)$. Having a positive family history for either cardiometabolic disease did not associate with a more adverse cardiometabolic risk profile (data not shown).

\section{Discussion}

This is the first study comparing cardiometabolic risk parameters in overweight children with and without T1DM. It is shown that overweight children with T1DM have lower waist circumference, higher levels of HDL cholesterol and lower levels of LDL cholesterol as compared to non-diabetic controls, but similar blood pressure and ALT values.

The difference between visceral fat (as measured by waist circumference) between overweight T1DM and nondiabetic children has not been described before, and the

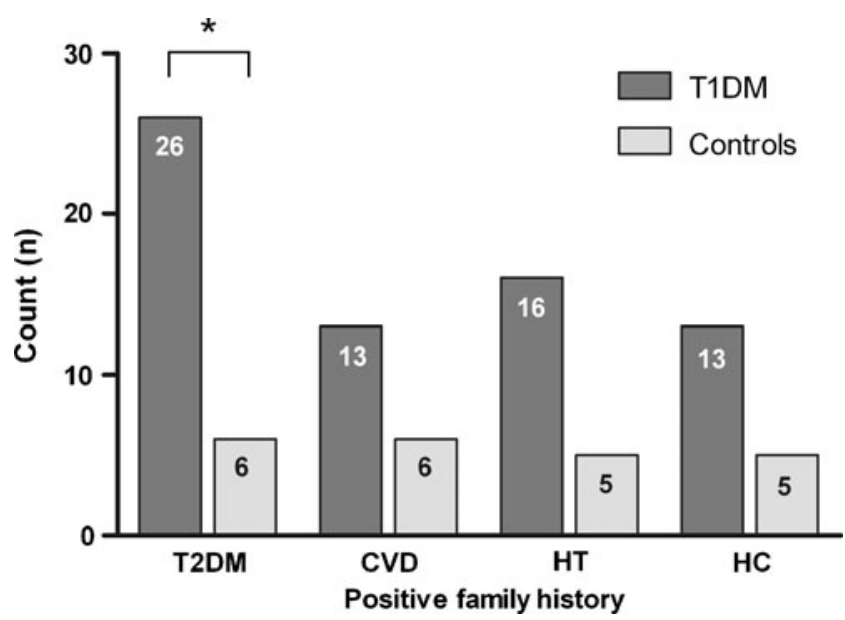

Fig. 1 Prevalence of a positive family history in overweight children with and without T1DM. ${ }^{*} P<0.001$. T2DM Type 2 diabetes mellitus, $C V D$ cardiovascular disease, $H T$ hypertension, $H C$ hypercolesterolaemia. Analysis with Fisher's exact test 
possible causes of this discrepancy remain speculative. Since the children were matched for sex, age, ethnicity and BMI, none of these factors are responsible. Moreover, the percentage of pubertal children was similar in both groups. Therefore, other factors, like diet, physical activity and the nature of overweight (i.e. due to well-controlled diabetes vs. a sedentary lifestyle), are likely to have caused the difference in body fat distribution. The differences in the nature of weight gain is substantiated by the finding that children with T1DM become more frequently overweight, but not obese, emphasising a different pathophysiological mechanism [9].

Part of the finding of a more favourable lipid profile in T1DM children as compared to non-diabetic children was already established in the SEARCH for Diabetes in Youth study, which reported higher HDL cholesterol levels (irrespective of glycaemic control) in children with diabetes as compared to their peers without diabetes [8]. In the same study, the suboptimal glycaemic control group showed higher total and LDL cholesterol levels, as compared to both the control and optimal glycaemic control group [8]. Glycaemic control is directly related to lipid metabolism, i.e. hypertriglyceridaemia is caused by a relative lack of circulating insulin, which results in unsuppressed lipolysis, a subsequent increase in free fatty acids, leading to an increased hepatic very LDL (VLDL) triglyceride output and an increased LDL cholesterol $[4,13]$. On the other hand, in well-controlled patients with T1DM, VLDL production is down-regulated due to augmented plasma insulin levels [20, 24].

In the present study, we found a majority of children to have a suboptimal glycaemic control and confirmed the association between suboptimal glycaemic control and an adverse lipid profile.

However, in spite of the large proportion of suboptimal controlled T1DM children, differences in lipid profiles between overweight children with and without T1DM become evident and may indicate an even greater difference between well-controlled overweight T1DM children and non-diabetic overweight controls.

The explanation for a more favourable lipid profile in T1DM children is probably also embedded in lifestyle differences and the nature of overweight (i.e. due to good glycaemic control). In line with the results of the Diabetes Control and Complications Trial, our previous study among 283 lean and overweight children with T1DM showed that overweight children with T1DM had higher blood pressure values, while having similar mean lipid levels in comparison to their peers of normal weight [22]. In contrast, in the same study, a trend was seen for adverse changes in lipid profile in obese children (Z-BMI, >2) as compared to normal weight children.

In the present study, we found high absolute numbers of a positive family history for cardiometabolic risk factors in the T1DM group. However, only the difference between a positive history for T2DM was significant between the T1DM and non-T1DM group. Since the included number of children is small, we are not able to compare this outcome to population based studies, such as the Bogalusa Heart study, which reported a positive family history for diabetes in $6 \%$ for normoglycaemic children and $9 \%$ for children with pre-diabetes [12]. The differences found (although mostly not significant) between the number of children having a positive family history between the T1DM and non-T1DM group may be due to a selection bias in the specialised diabetes clinic. It may well be that individuals familiar with the medical circuit (i.e. in families of children with a positive family history) are more focused on specialised care for their children.

There is a lack of studies investigating the difference for cardiometabolic outcomes between overweight populations with and without T1DM. A recent study by Franks et al., among a cohort of 4,857 American Indian non-diabetic children, with a mean follow-up of 23.9 years, showed that total cholesterol levels were not associated with premature death of endogenous causes, whereas for BMI, blood pressure and glucose intolerance, strong associations were found [5]. However, in the same study, other lipid levels (triglycerides, HDL cholesterol and LDL cholesterol) were not determined, and therefore, no conclusions could be drawn with respect to these measures and cardiometabolic outcome. In conclusion, since the role of lipid abnormalities in youth without diabetes in predicting cardiometabolic health outcome remains unknown, no extrapolations can be made towards specific populations (i.e. overweight individuals with T1DM) between the association of lipid profile and actual cardiometabolic risk.

Some limitations of this study should be mentioned. First of all, the study was mostly underpowered for detecting between group differences. In fact, our study results showed smaller values for the correlation of LDL and HDL cholesterol leading to a power of $65-70 \%$ and thus the requirement of a larger sample size. Despite this fact, we were still able to show differences between groups. Therefore, the true differences are expected to be even larger than presented in our study. Another limitation is that lipid levels were measured non-fasting in children with T1DM, a challenge that was overcome by recalculating nonfasting lipid total cholesterol, triglyceride levels and LDL cholesterol. We believe that the potential bias introduced by this method is minimal, since differences for total and LDL cholesterol were already found also without recalculation of non-fasting lipid levels. In addition, the study by Margeirsdottir et al. found no difference between fasting and non-fasting lipid levels for HDL and LDL cholesterol levels in 50 children with T1DM in whom they tested both [10]. The strength of this study is the study design, which enables to provide outcomes that are unlikely to be biased by 
the factors matched for (ethnicity, age, Z-BMI and sex). However, a limitation may be that additional bias could have occurred with Z-BMI, since children with a wide range of ZBMI were included and different results may have been found when using patients with a Z-BMI at one end of the spectrum. Additionally, the study design is not suitable for determining the effect of increasing BMI on the difference in lipid values between the two study groups. Therefore, this study is not capable of predicting the difference in lipid profiles between children with- and without T1DM with increasing BMI values. Finally, since Tanner stages were not recorded, we cannot be certain of an equal number of pubertal children in both groups, which might be partly responsible for the difference in Z-WC found between T1DM and non-T1DM children. However, since children were matched on age, ethnicity and sex, a significant difference would be unlikely.

In conclusion, overweight children with T1DM show a more favourable cardiometabolic risk profile as compared to children without T1DM. Moreover, family history for cardiometabolic outcomes was more prevalent among children with T1DM as compared to non-diabetic children. Longitudinal studies among overweight paediatric cohorts (with and without T1DM) are needed to quantify actual differences for cardiometabolic health between these groups in later life.

Open Access This article is distributed under the terms of the Creative Commons Attribution Noncommercial License which permits any noncommercial use, distribution, and reproduction in any medium, provided the original author(s) and source are credited.

\section{References}

1. (2001) Influence of intensive diabetes treatment on body weight and composition of adults with type 1 diabetes in the Diabetes Control and Complications Trial. Diabetes Care 24:1711-1721

2. American Diabetes Association (2006) Standards of medical care in diabetes-2006. Diabetes Care 29(Suppl 1):S4-S42

3. Dietz WH (1998) Health consequences of obesity in youth: childhood predictors of adult disease. Pediatrics 101:518-525

4. Dullaart RP (1995) Plasma lipoprotein abnormalities in type 1 (insulin-dependent) diabetes mellitus. Neth J Med 46:44-54

5. Franks PW, Hanson RL, Knowler WC, Sievers ML, Bennett PH, Looker HC (2010) Childhood obesity, other cardiovascular risk factors, and premature death. N Engl J Med 362:485-493

6. Fredriks AM, van Buuren S, Wit JM, Verloove-Vanhorick SP (2000) Body index measurements in 1996-7 compared with 1980. Arch Dis Child 82:107-112

7. Friedewald WT, Levy RI, Fredrickson DS (1972) Estimation of the concentration of low-density lipoprotein cholesterol in plasma, without use of the preparative ultracentrifuge. Clin Chem 18:499-502

8. Guy J, Ogden L, Wadwa RP, Hamman RF, Mayer-Davis EJ, Liese AD, D'Agostino R Jr, Marcovina S, Dabelea D (2009) Lipid and lipoprotein profiles in youth with and without type 1 diabetes: the
SEARCH for Diabetes in Youth case-control study. Diabetes Care 32:416-420

9. Liu LL, Lawrence JM, Davis C, Liese AD, Pettitt DJ, Pihoker C, Dabelea D, Hamman R, Waitzfelder B, Kahn HS (2009) Prevalence of overweight and obesity in youth with diabetes in USA: the SEARCH for Diabetes in Youth Study. Pediatr Diabetes 11:4-11

10. Margeirsdottir HD, Larsen JR, Brunborg C, Overby NC, hlJorgensen K (2008) High prevalence of cardiovascular risk factors in children and adolescents with type 1 diabetes: a populationbased study. Diabetologia 51:554-561

11. Must A, Jacques PF, Dallal GE, Bajema CJ, Dietz WH (1992) Long-term morbidity and mortality of overweight adolescents. A follow-up of the Harvard Growth Study of 1922 to 1935. N Engl J Med 327:1350-1355

12. Nguyen QM, Srinivasan SR, Xu JH, Chen W, Berenson GS (2009) Influence of childhood parental history of type 2 diabetes on the pre-diabetic and diabetic status in adulthood: the Bogalusa Heart Study. Eur J Epidemiol 24:537-539

13. Nikkila EA, Kekki M (1973) Plasma triglyceride transport kinetics in diabetes mellitus. Metabolism 22:1-22

14. Purnell JQ, Hokanson JE, Marcovina SM, Steffes MW, Cleary PA, Brunzell JD (1998) Effect of excessive weight gain with intensive therapy of type 1 diabetes on lipid levels and blood pressure: results from the DCCT. Diabetes Control and Complications Trial. JAMA 280:140-146

15. Schwab KO, Doerfer J, Krebs A, Krebs K, Schorb E, Hallermann K, Superti-Furga A, Zieger B, Marz W, Schmidt-Trucksass A, Winkler K (2007) Early atherosclerosis in childhood type 1 diabetes: role of raised systolic blood pressure in the absence of dyslipidaemia. Eur J Pediatr 166:541-548

16. Schwab KO, Doerfer J, Naeke A, Rohrer T, Wiemann D, Marg W, Hofer SE, Holl RW (2009) Influence of food intake, age, gender, $\mathrm{HbA1c}$, and BMI levels on plasma cholesterol in 29,979 children and adolescents with type 1 diabetes-reference data from the German diabetes documentation and quality management system (DPV). Pediatr Diabetes 10:184-192

17. Sibal L, Neely RD, Jones A, Home PD (2010) Friedewald equation underestimates low-density lipoprotein cholesterol at low concentrations in young people with and without type 1 diabetes. Diabet Med 27:37-45

18. Silverstein J, Klingensmith G, Copeland K, Plotnick L, Kaufman F, Laffel L, Deeb L, Grey M, Anderson B, Holzmeister LA, Clark N (2005) Care of children and adolescents with type 1 diabetes: a statement of the American Diabetes Association. Diabetes Care 28:186-212

19. Strauss RS, Pollack HA (2001) Epidemic increase in childhood overweight, 1986-1998. JAMA 286:2845-2848

20. Taskinen MR (1992) Quantitative and qualitative lipoprotein abnormalities in diabetes mellitus. Diabetes 41(Suppl 2):1217

21. van Vliet M, von Rosenstiel I, Schindhelm RK, Brandjes DP, Beijnen JH, Diamant M (2009) Ethnic differences in cardiometabolic risk profile in an overweight/obese paediatric cohort in The Netherlands: a cross-sectional study. Cardiovasc Diabetol 8:2

22. van Vliet M, Van der Heyden JC, Diamant M, Schindhelm RK, von Rosenstiel I, Aanstoot HJ, Veeze HJ (2010) Overweight is highly prevalent in children with type 1 diabetes and associates with cardiometabolic risk. J Pediatr 156:923-929

23. Weiss R, Dziura J, Burgert TS, Tamborlane WV, Taksali SE, Yeckel CW, Allen K, Lopes M, Savoye M, Morrison J, Sherwin RS, Caprio S (2004) Obesity and the metabolic syndrome in children and adolescents. N Engl J Med 350:2362-2374

24. Winocour PH, Durrington PN, Ishola M, Anderson DC (1986) Lipoprotein abnormalities in insulin-dependent diabetes mellitus. Lancet 1:1176-1178 